\title{
Modeling and Simulation of Hall Voltage Sensor Based on Physical Modeling Environment
}

\author{
Chunming $\mathrm{Li}^{1, \mathrm{a}}$, Yan $\mathrm{Xu}^{2, \mathrm{~b}}$ and Tian $\mathrm{Ma}^{3, \mathrm{c}}$ \\ ${ }^{1}$ China North Vehicle Research Institute, No.4, Huaishuling, Fengtai, Beijing, China \\ ${ }^{2}$ China North Vehicle Research Institute, No.4, Huaishuling, Fengtai, Beijing, China \\ ${ }^{3}$ China North Vehicle Research Institute, No.4, Huaishuling, Fengtai, Beijing, China

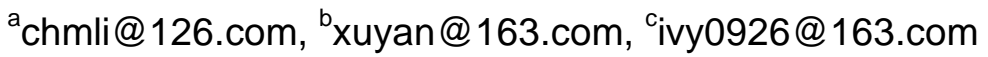

\begin{abstract}
Keywords: Hall Voltage Sensor, Hall Effect, Simulation Model
Abstract. Hall voltage sensor is one of the most widely used sensors in the field of today's electronic measurement. It can completely replace the traditional transformer and shunt, and with the advantages of high precision, good linearity, wide frequency band, fast response, strong overload capacity and without losing the energy of measurement circuit, etc. This article by MATLAB / simulink simulation tool establishes under a Simscape modeling environment can reflect the hall voltage sensor measurement principle, internal mechanism and the measurement error of the simulation model. The model reflects the principle and internal structure of the closed-loop hall voltage sensor, introduces the interference terms such as noise, and the model has no load effect on the measuring circuit, so that the simulation system can better reflect the actual situation.
\end{abstract}

\section{Introduction}

In recent years, a large number of new generation of power semiconductor devices enter the fields of power electronics, AC frequency control of motor speed, inverter device and switch power supply, etc. Hall voltage sensor is developed in recent years measuring the control voltage of a new generation of industrial electricity sensor. It is a new type of high-performance electrical detection element ${ }^{[1]}$.

Hall voltage sensor has the excellent electrical performances of wide bandwidth, short response time, strong anti-interference ability, high original side and secondary side of insulation voltage ${ }^{[2]}$. Using the same hall voltage sensor test component can only test AC and DC, even can test transient peak, so that it is an alternative to transformer and shunt new generation of products ${ }^{[3]}$.

This article establishes under a Simscape physical modeling environment of closed-loop hall voltage sensor of simulation model. The model reflects the principle and internal structure of closed-loop hall voltage sensor. The measurement result contains an error of Gaussian distribution. The error of the mean and variance can be set by the parameter interface. The model has no load effect on the measuring circuit.

\section{Summary of hall voltage sensor}

Model of the sensor based on hall effect, established a zero-flux hall effect current sensor model, as shown in figure 1 . The model had no effect on the original edge circuit (no tap, no pressure, no energy drain), by setting the output voltage of hall drift consider measurement error and noise ${ }^{[4,5,6]}$. The output of the model of 0 to $5 \mathrm{v}$ voltage.

The mechanism diagram of hall sensor is shown in figure 1 below. 


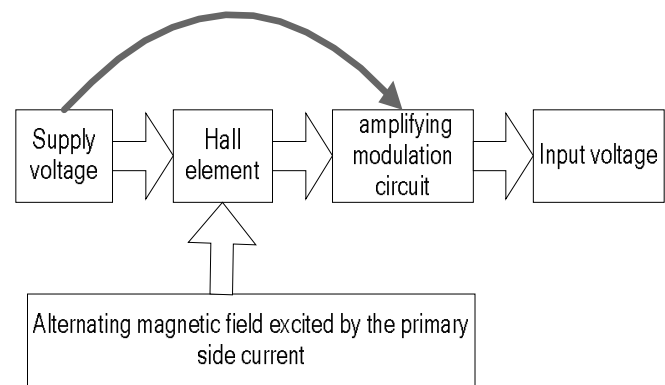

Fig. 1. Hall voltage sensor structure diagram

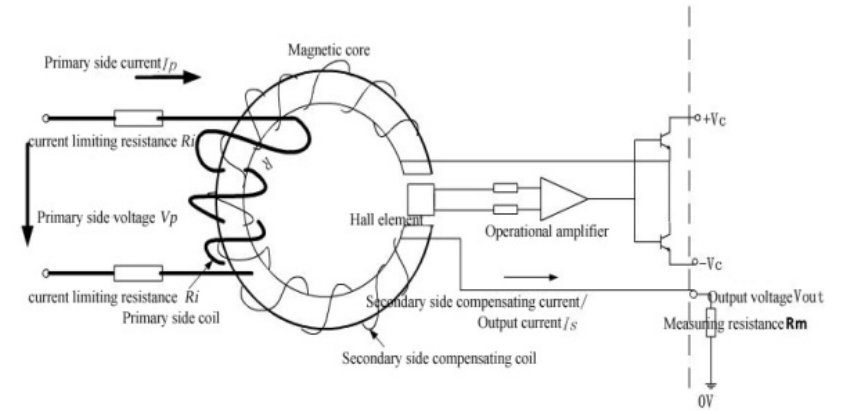

Fig. 2. The principle diagram of the hall voltage sensor

\section{Model principle}

Shown in figure 2 the magnetic balanced hall voltage sensor working principle is: the original voltage through the side resistance $R_{i}$ produced the original current $I_{p}^{\prime}$,

$$
I_{P}^{\prime}=\frac{V_{p}}{2 R_{i}} .
$$

Primary side of coil magnetic field B and controlled by the size of the hall voltage compensation current flows $I_{s}^{\prime}$ through the vice edge instead of magnetic fields produced by compensating coil compensation, its compensation current $I_{s}^{\prime}$ accurately reflect the size of the primary side voltage $V_{p}$,

$I_{s}^{\prime} \bullet N_{s}=I_{p}^{\prime} \bullet N_{p} \cdot$

According to the type (1) with type (2), the original side voltage,

$V_{p}=2 R_{i} I_{p}^{\prime}=2 R_{i} \cdot \frac{I_{s}^{\prime} \bullet N_{s}}{N_{p}} \cdot$

(3)

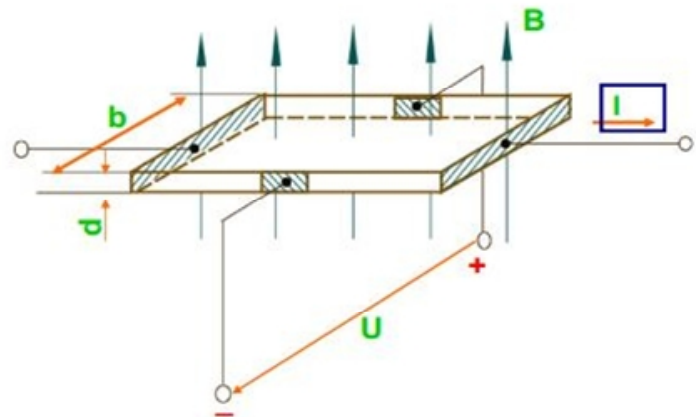

Fig. 3. Hall film

Hall film

In figure 3, a rectangular semiconductor through magnetic induction intensity of B. Control current in a pair of side keys by $i$. The electric charge of the angle between the direction of motion and the direction of the magnetic field is influenced by the Lorenz force and lead to the carrier direction perpendicular to the current direction and the magnetic field direction of the side of the aggregation ${ }^{[7]}$. The voltage is established in the other side. Hall element achieve the magnetic field convert to the hall voltage.

\section{Measurement error}

In the hall element module, the outside of the two magnetic circuit ports and the two circuit ports are 2 input terminals, respectively the output hall voltage drift and noise of hall film.

If the measurement error caused by $U_{D}$ is $\Delta I$, there is ${ }^{[8]}$ 
$U_{D}=\Delta \times \frac{K_{H} I_{\text {cantro }} N_{1} S_{\text {core }} \mu_{0}}{S_{\text {hall }}\left(L_{\text {ron }} / \mu+L_{\text {air }}\right)}$.

In the process of using, users only need input $\Delta I$ value, in the model automatically.

$U_{D}$ is the hall film bias voltage.

\section{Simulation verification}

To test the availability of modules by building the model as follows.

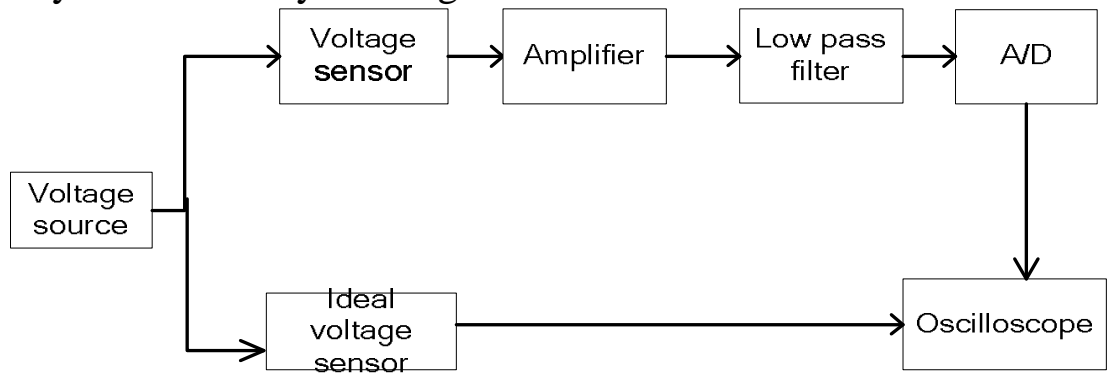

Fig. 4. Example simulation system diagram

Figure 4 shows that the system is connected by an AC voltage source, a resistor, an ideal voltage meter, and a voltage sensor model and Good circuit reference and solution. The parameters setting of the simulation system: Power supply voltage amplitude is $400 \mathrm{~V}$, Resistance is $1 \Omega$, Default parameters are used in the model.

The results are shown in Figure 5 (a) and (b). Can see the measurement result have a certain range of fluctuations in the actual results .

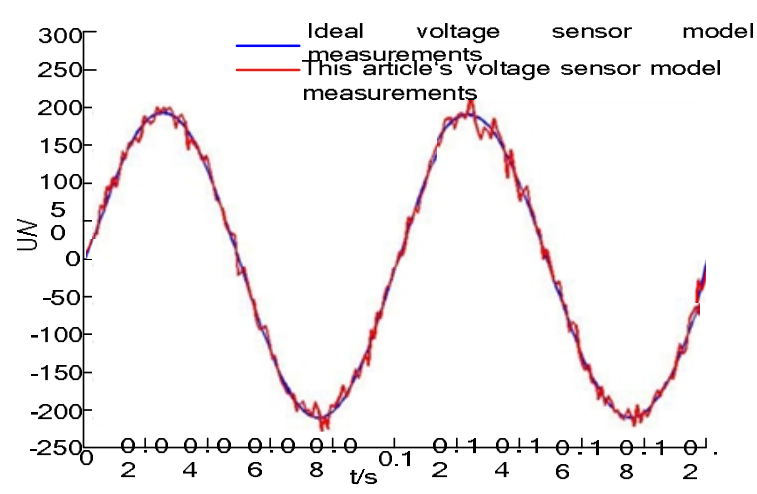

(a)

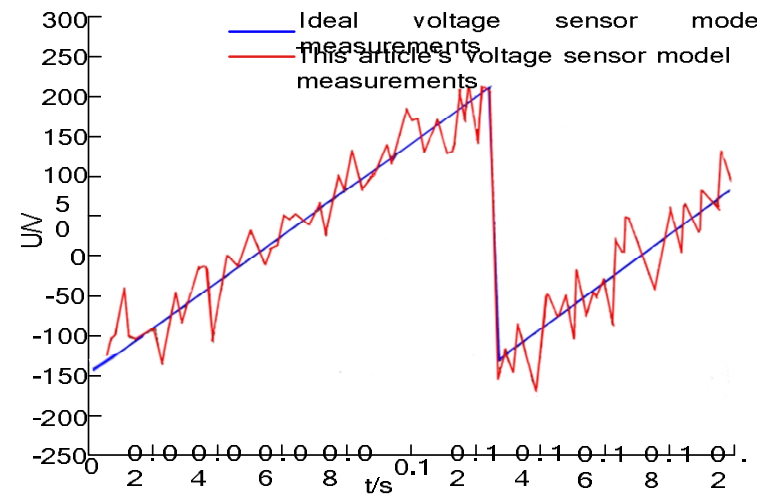

(b)

Fig. 5. Comparison of measurement results

\section{Conclusion}

In this paper, by using MATLAB/simulink to establish the hall voltage sensor model simulation in simscape. From the simulation results, the simulation model has good measurement results which reflect the measurement error and the error size can be adjusted according to the needs, suitable for most of the waveform of the voltage measurement, can fully meet the requirements of the design of the sensor.

\section{Acknowledgements}

This paper was financially supported by Science and Technology On Vehicle Transmission Laboratory Fund. 


\section{References}

[1] TogawaK, SanbonsugiH and LapickiA: High-sensitivity InSb thin-film micro-Hall sensor arrays for simultaneous multiple detection of magnetic beads for biomedical applications[J].IEEE Transactions on Magnetics 2005,41(10):3661 - 3663 .

[2] Bai Shaohong,Development of Integrated Hall Transducer[J]. Automatic instrument, 2003,24(3):1-9

[3] Li Fuan,The design of closed loop hall current sensor and testing[D].Huazhong University of Science and Technology,2013.

[4] Edward Ramsden.Hall-Effect Sensors Theory and Applications[M].newnes,2006,50-100

[5] F.Nguyen Van Dau, A.Schuhl, J.R.Childress and M.Sussiau , "Magnetic sensors for nanotesla detection using planar Hall effect" , IEEE the 8thInternational Conference on Solid-State Sensors and Actuators, and Eurosensor IX,Stockholm, Sweden, pp241-244, June 1995.

[6] Feng Ming, Research and development of Holzer phase sensor[D],Shang Hai: Shanghai Institute of micro system and information technology, Chinese Academy of Sciences, 2004

[7] Dou Qiaoqi, Dot Holzer current sensor, Chinese utility model patent, ZL200820065435.4.

[8] M. Pastre , M. Kayal , and H. Blanchard, Continuously calibrated magnetic field sensor. European Patent 1637898 , March 2006. 